\title{
Occurrence and Antimicrobial Susceptibility Pattern of Bacteria Isolated from Gastrointestinal Tract of Fresh Water Fishes in Abuja, Nigeria
}

\author{
Mailafia Samuel $^{1 *}$ and Anjorin Samuel Toba ${ }^{2}$ \\ ${ }^{1}$ Department of Veterinary Microbiology, Faculty of Veterinary Medicine, \\ University of Abuja, Nigeria \\ ${ }^{2}$ Department of crop science, Faculty of Agriculture, University of Abuja, Nigeria
}

*Corresponding author

\begin{tabular}{|c|c|}
\hline & A B S T R A C T \\
\hline & \multirow{7}{*}{$\begin{array}{l}\text { Bacterial microflora of fishes is part of a complex ecosystem responsible for a variety of } \\
\text { diseases in fish and man. A survey was conducted to determine the occurrence and } \\
\text { antimicrobial susceptibility of microorganisms from the gastrointestinal tract of } 220 \text { fishes } \\
\text { belonging to two specie Clarias gariepinus and Heterobranchus species. A total of } 5 \\
\text { bacterial species were identified and their prevalences were: Escherichia coli } 16(36.60 \%) \text {, } \\
\text { Proteus vulgaris } 10(22.70 \%) \text { Salmonella typhi } 4(9.09 \%) \text {. Staphylococcus aureus } 8 \\
(18.80 \%) \text { and Staphylococcus epidermidis } 6(13.63 \%) \text {. Antibiotic susceptibility by } \\
\text { differential standardized disc method showed high incidence of resistance to } \\
\text { cotrimoxazole, streptomycin and tetracycline as well as a low resistance to ciprofloxacin, } \\
\text { sparfloxacin and pefloxacin by the isolated organisms. Statistical analysis showed that } \\
\text { there was significant positive association between the prevalence of isolates and their } \\
\text { susceptibility to the various antibiotics }\left(\mathrm{X}^{2}=72.12 \text {; p }<0.05 \text { and p=0.00). This findings }\right. \\
\text { dissipated array of microbial isolates and the sensitivity and resistant patterns of the isolates } \\
\text { to a variety of antimicrobial agents. The difference in the sensitivity of the isolates to a } \\
\text { variety of antibiotics as observed in this study could be attributed to strain or specie } \\
\text { differences, and also the usage, misuse or abuse of these drugs coupled with prolonged } \\
\text { antibiotic therapy which has favored the emergence of resistant strains. There is need for } \\
\text { rational approach in monitoring of microorganisms and their sensitivities to control these } \\
\text { diseases in the human population. }\end{array}$} \\
\hline Keywords & \\
\hline & \\
\hline $\begin{array}{l}\text { Fresh water fishes, } \\
\text { GIT, Bacteria. }\end{array}$ & \\
\hline Article Info & \\
\hline $\begin{array}{l}\text { Accepted: } \\
\text { 25 February } 2017 \\
\text { Available Online: } \\
10 \text { April } 2017\end{array}$ & \\
\hline & \\
\hline
\end{tabular}

\section{Introduction}

Bacteria of fish are closely associated with one another of particular interest do those inhabit the gastrointestinal tract (GIT). These microorganisms enter the intestinal tract of fish around the time of first feeding, and the microorganism becomes established to cause infection in different organs of the fish (Bauer et al., 1996; Ben Khemis et al., 2003; Bergey, 1992; Birkbeck et al., 2002). Microbial composition can be affected by bacterial load and composition of the ambient water as well as diet (Ben Khemis et al., 2003; Cheesbrough, 2005). Other factors such as the development of the digestive tract and temperature can also alter the intestinal microbiology. It is believed that intestinal microorganisms established during the larval stage will develop into a persistent flora in 
juvenile and adult fish (Hansen et al., 1999). The beneficial effects of the intestinal microbiology to fish might include protecting the fish against pathogens by preventing the pathogens from colonizing the intestinal tract and aiding in fish nutrition by contributing enzymes and micronutrients (Ringo et al., 1990).

Disease is a major problem in the fish farming industry and there is a risk associated with the transmission of resistant bacteria from aquaculture environments to humans, and risk associated with the introduction in the human environment of nonpathogenic bacteria, containing antimicrobial resistance genes, and the subsequent transfer of such genes to human pathogens (FAO, 2007; Collinder et al., 2003). Understanding the composition of the intestinal microbes and their roles in fish can help increase the success rate of fish culture. With that knowledge, aqua culturists and researchers can have basis for monitoring and controlling the intestinal infections to aid in higher survival rates of marine fish (Huber et al., 2004).

Antibiotics inhibits or kill beneficial microbiota in the gastrointestinal ecosystem but it also made antibiotic residue accumulated in fish products to be harmful for human consumption (WHO, 2006). The European Union has therefore ratified a ban for the use of all sub-therapeutic antibiotics as growth-promoting agents in aqua cultural practices. In our study, the microbial ecology inhabiting the GIT of two fresh water fishes has been investigated. There are several documented evidence that proved that the alimentary tract of fish consist of a complex ecosystem, containing large number of microorganisms (Spanggaard et al., 2000). Microbial populations in the intestinal contents are much higher than those in the surrounding water. It is known from studies of the intestinal micro flora of fishes that the resident bacterial population of the intestine influences the establishment of host pathogenicity due to favorable ecological niches for microbial proliferation (Gomathi et $a l ., 2016)$. Therefore, early identification and institution of appropriate treatment is necessary to reduce the morbidity and mortality due to the organisms in fish (13). However, the main objectives of the study were to identify the microorganisms prevalent in the GIT of fresh water fishes and to identify their susceptibility to commonly used antimicrobial agents. The findings will add to current knowledge of microbial ecology of the gastrointestinal tract of fishes in Nigeria.

\section{Materials and Methods}

\section{Study Area}

The research work was carried out in Microbiology Laboratory of the Department of biological science, University of Abuja, Gwagwalada, Nigeria. Abuja is the capital territory of Nigeria. The territory is centrally located and covers a wide area of land of about 8000 square. It is an 8,000 square kilometer land area centrally located and bound on the north by Kaduna State, on the east by Nassarawa State, on the west by Niger state and on the south/west by Kogi State. It lies between latitude $8.25^{\circ}$ and $9.2^{\circ}$ north of the equator and longitude 6.45 and 7.39 east of the Greenwich Meridian. Abuja is geographically located in the nerve center of Nigeria (Ben et al., 2003; Olafsen, 2001).

\section{Collection of Samples and processing}

Two hundred and twenty fishes samples from two different species (Clarias gariepinus in which 110 samples were collected) and Heterobranchus species in which 110 samples were also collected) were collected from different ponds at the agricultural development programme (ADP) Phase 2 
Gwagwalada. The samples were carefully transported in ice-packed containers to the microbiology laboratory in the Department of Biological Sciences, University of Abuja for analysis.

The number of incidental organism was reduced by washing fish skin with $70 \%$ ethanol. Then the ventral surface was opened with sterile scissors. After dissecting the fish the intestinal tract of the fish content was removed and macerated in a mortar. A sterile swab sticks were removed from the seal and carefully used to make a swab of the macerated fish intestine in the mortar so as to collect small fluids that contains organisms that may be found in the gastrointestinal tract of fish. The swab sticks were carefully placed into test tubes containing already prepared and sterilized nutrient broth and covered quickly. The same procedure is repeated for all other samples and then labeled respectively (Cheesbrough, 2005).

\section{Laboratory Culture and Identification}

The inoculated test tubes were incubated at $37^{\circ}$ Cfor 24hours and then observed for microbial growth. Appropriate quantity of selective media such as nutrient agar, MacConkey's agar, Mannitol salt agar and Sabouraud's dextrose agar was prepared into a conical flask, packed and sterilized in an autoclave for $20 \mathrm{mins}$ at $121^{\circ} \mathrm{C}$. After autoclaving, the media is then removed from the autoclave and carefully poured in petri dishes as many as required and gently covered and allowed to cool and solidify. A full loop of the organism in the test tubes was collected using an inoculating loopand streaked on the four different selective media (Macconkay agar. Manitol salt agar, Sabouraud's dextrose agar, and nutrient agar) and incubated at $37^{\circ} \mathrm{C}$ for 24 hours. Microbial colony counts were taken using digital colony counter after incubation for the identified bacteria and fungi species. The pure cultures of isolates were preserved on nutrient agar plates and stored on agar slants at $4^{0} \mathrm{C}$. The pure isolates were characterized on the basis of grams staining/microscopy, biochemical tests and sensitivity test. The biochemical tests did include: catalase, oxidase test, indole test, and triple sugar ion test, DNA's test, gelatin liquefaction, esculin hydrolysis, methyl red test, vogues proskraver test, citrate utilization test, urease test, SIM tests, coagulase, Simmons citrate, esculin and fermentation of sugars such as: salicin, sucrose, glucose, mannitol, galactose (Ben Khemis et al., 2003; Bergey, 1992).

\section{Antibiotic Susceptibility Test}

Antibiotic susceptibility test of the isolates against commonly prescribed antibiotics was determined using the standard microbiological protocol by the Kirby - Bauer method. The standard antibiotic molto discs used where those of maxidics ${ }^{\mathrm{R}}$ (Enugu, Nigeria) which included cotrimoxazole $(20 \mathrm{mcg})$, gentamicin (10mcg), amoxicillin (30mcg), sparfloxacillin (30mcg), Ofloxacin (30mcg), cloramphenicol (10mcg), streptomycin (15mcg), tetracycline $(25 \mathrm{mcg})$, ciprofloxacin $(5 \mathrm{mcg})$ and pefloxacin (30mcg).18 h culture of each isolate was prepared by dislodging a small portion of the test isolates into $2 \mathrm{mls}$ of already sterilized peptone water in sterile test tubes and was shaken vigorously to disperse the cells in the peptone water. The test tubes were then incubated overnight and for $18 \mathrm{~h}$. After incubation the milky suspensions were then used to seed the Muller Hinton agar at room temperature by aseptically transferring $2 \mathrm{ml}$ of each represented isolates into the agar. The agar plates were swirled to dispense cells and the excess suspension was decanted close to a fire source aseptically. The plates were left for about 30 min to allow the proper diffusion of the antibiotics. The standard antibiotic sensitivity disc were then aseptically placed at 
the centre of the seeded Mueller Hinton agar (in duplicates), and allowed to stand for 30 minutes. The plates were then incubated at $37^{\circ} \mathrm{C}$ for $18 \mathrm{~h}$ aerobically. The diameter of the zones of inhibition produced by each antibiotics on the disc were measured using a meter rule and the result recorded in millimeters and interpreted as either susceptible (s) or resistance (r) to the antibiotic agent used, depending on the length of zone diameter of inhibition produced compared to reported standard length: $0-5 \mathrm{~mm}$ regarded as resistance, $(\mathrm{R}), 5-15 \mathrm{~mm}$ sensitive, $\left(\mathrm{S}_{1}\right)$ 16-25mm $\left(\mathrm{S}_{11}\right)$ and $26-35 \mathrm{~mm}\left(\mathrm{~S}_{111}\right)^{(1,19)}$. Statistical analysis was carried out using Chisquare test to attain a Pearson CM-square value as described by (Bauer et al., 1996).

\section{Results and Discussion}

All the fishes specimen examined were positive for microorganisms. Five bacterial genera where identified from the gastrointestinal tract of fresh water fish. Among the gram negative organisms isolated includes E. coli, P. vulgaris and Salmonella typhi. The gram positive bacterial genera isolated are Staphylococcus aureus and S.epidermidis. Out of the 44 bacterial isolates from the gastrointestinal tract of fish $36.6 \%$ (16 isolates) were E. coli, $22.7 \%$ (10 isolates) were $P$. vulgaris, $9.09 \%$ (4 isolates) were Salmonella typhi, $18.80 \%$ (8 isolates) were Staphylococcus aureus and $13.63 \% \quad$ (6 isolates) were Staphylococcus epidermidis. This indicated that E. coli occurred most followed by $P$. vulgaris, $S$. aureus, S.epidermidis and Salmonella typhi respectively. The statistical analysis showed that there is significant difference between the isolates and antibiotics $\left(x^{2}=72.12 ; P<0.05\right.$ and $P=0.00)$. This indicates that there is positive association of the isolates to different isolation sites. Table 2 shows the morphological characteristics of the bacterial isolates on culture plates. Morphological characteristics of these isolates on culture plate showed that
E. coli showed pink coloration on MacConkey agar plate with opaque appearance. $P$. vulgaris showed brown coloration on MacConkey agar plate with opaque appearance. S. typhi showed black coloration on salmonella-shigella agar (SSA) plate with opaque appearance. S.aureus showed yellow coloration on manitol salt agar plate with translucent appearance. S.epidermidis showed pink coloration on manitol salt agar plate with opaque appearance. Table 3 shows the biochemical reactions of the various isolates to different tests for example, E.coliwas positive to indole, catalase and produce gas with yellow slant; $P$. vulgaris were positive to urease, indole and produces hydrogen sulphide etcetera.

Table 4 shows dissipation of antimicrobial susceptibility of the gram negative organisms tested. E.coli was resistant to septrin and streptomycin but showed low sensitivity to tarivid and chloramphenicol, moderate sensitivity to amoxicillin and tetracycline and high sensitivity to ciprofloxacin, pefloxacin, sparfloxacin and gentamycin. $P$. vulgaris showed resistant to streptomycin, septrin, gentamycin, chloramphenicol and amoxicillin, low sensitivity to tarivid, sparfloxacin and tetracycline and moderate sensitivity to ciprofloxacin and pefloxacin. S.typhi showed resistance to tetracycline, streptomycin and cotrimoxazole, moderate sensitivity to ofloxacin, chloramphenicol, and amoxicillin and high sensitivity to ciprofloxacin, pefloxacin, sparfloxacin and gentamycin. $S$. aureus showed resistant to amoxicillin, ampicillin and ampiclox, low sensitivity to erythromycin, streptomycin and tetracycline and high sensitivity to to amikacin, ciprofloxacin, sparfloxacin and gentamycin and $S$. epidermidis showed resistant to amoxicillin, ampiclox and ampicillin, low sensitivity to erythromycin, streptomycin and tetracycline, high sensitivity to gentamycin, amikacin and ciprofloxacin and to sparfloxacin. 
The susceptibility testing of isolates were studied and the interpretation of zones of inhibition was determined according to zone size of chart of Kirby - bauer test. Antibiotic susceptibility profiles showed that Ciprofloxacin, pefloxacin, sparfloxacin and gentamycin appeared to be the most efficient antibiotics for $E$. coli as shown by its zones of inhibition. Ciprofloxacin and pefloxacin are the most efficient antibiotics for Proteus vulgaris. Ciprofloxacin, pefloxacin, sparfloxacin and gentamycin are the most efficient for S. typhi. Gentamycin, sparloxacin, amikacin and ciprofloxacin are most efficient for $S$. aureus while sparfloxacin is the best for S. epidermidis. In general, ciprofloxacin and sparfloxacin are the most efficient antibiotics for the different group of isolates as indicated by their zones of inhibition.

Table 5 shows antibiotic resistant patterns of the isolates from fishes. A total of 8 different antibiotics were not susceptible to all the bacterial species isolated. 2 antibiotics (STM and SXT) ad resistant to E. coli, 3 antibiotics (STM, SXT and TET) were resistant to S.typhi, 4 antibiotics (STM, SXT, GN and $\mathrm{CH})$ were resistant to Proteus vulgaris, 3 antibiotics (AMP, APX and AM), were resistant to S.aureus and 3 antibiotics (AMP, APX and AM) were resistant to S.epidermidis.

This study has shown that the gastrointestinal tract of fresh water fish habours bactrerial organisms such as E. coli, Proteus vulgaris, Salmonella typhi, Staphylococcus aureus, Staphylococcus epidermidis. These agrees with the findings from other similar studies and suggests that Enterobacteriaceae especially the coliforms are relatively the leading organism in the gastrointestinal tract of fresh water fish. This may be due to the fact that the fishes are exposed to some common source of contamination which may be through faecal contaminated water source, contaminated feed and environment where the fishes are cultured (Olafsen, 2001).
The high incidence of Enterobacteriaceae recorded in this study could be due to the virulent factors present within these organisms which gives them the ability to be resistant to antibiotics. The result of these work also agree perfectly with the similar result carried out by (Olayemi et al., 1997) were as high as $45.3 \%$ incidence of Enterobacteriaceae among other organisms were recorded in Gombe state in Nigeria. Similarly E. coli was also incriminated as the highest organism (36.6\%) that was isolated from the gastrointestinal tract of fresh water fish as reported (Trust, 1974). In this work three gram negative organisms (E. coli, Proteus vulgaris, Salmonella typhi) were isolated while two gram positive organisms (Staphylococcus aureus and Staphylococcus epidermidis) were also isolated.

The incidence of S.aureus and S.epidermidis in the gastrointestinal tract of fresh water fish may be due to contamination from the skin of individuals handling the fish culture. Since S.aureus can be found on human skin and S.epidermidis is a normal flora of the skin it can be easily transferred to the fish culture through feeding and water source (Ikegwu et al., 2008). The findings here confirm that fish can be infected with varieties of microbial species, especially those bacteria in fresh water environment. It has also been established that these microflora of fishes are a function of the micro flora of the environment as indicated by the similarities between the isolates and the typical fresh water bacteria. However, most of the isolates identified as members of Enterobacteriaceae particularly coli forms are associated with fecal contamination and are also indicative of the possible presence of enteric pathogens. Therefore the isolates potentiates serious consequences to their host (fishes) to animals that feed on them and finally to man. The microbial population constitutes a significant burden throughout the life span of fishes and it 
has a role in nutrition, growth and disease susceptibility (Kanika, 2007). For a better decision - making, physicians need more information about local susceptibility patterns of these microorganisms isolated. Therefore it is a rational approach to perform microbiological examination of these microorganisms in the GIT of fresh water fishes along with their antibiogram to assess the trend of antibiogram of GIT microorganisms in any fresh water environment. The difference in the sensitivity pattern of the isolates to different antibiotics as observed in this study could be attributed to strain differentiation, geographic location, misuse and abuse of drugs and prolonged use of some of these antibiotics which has favored the emergence of resistant strains. Therefore there is need to constantly monitor susceptibility patterns of this microflora isolated and the commonly used antimicrobial susceptibility agents, as these will help to check the emergence of resistant strains. The sensitivity patterns of Enterobacteriaceae species (E. coli, Proteus vulgaris and Salmonella typhi) to antibiotics recently reported showed that these organisms dissipated high frequency of multiple antibiotic resistance which is similar to the study carried out on antimicrobial susceptibility pattern of enteric bacteria. It was further indicated from our findings that the bacteria was highly sensitive to ciprofloxacin, pefloxacin and sparfloxacin while high resistance were recorded against septrin and streptomycin. Also in the study carried out on antimicrobial susceptibility pattern of S.aureus in Jos Plateau State Nigeria were found to be highly sensitive to amoxicillin, ciprofloxacin, sparfloxacin and gentamycin while high resistance was recorded against amoxicillin, ampicillin and ampiclox (Evans et al., 2007; Trust et al., 1974). It was reported that Staphylococcus epidermidis was highly sensitive to gentamicin, amoxicillin, ciprofloxacin and sparfloxacin while high resistance was recorded against amoxicillin, ampiclox and ampicillin.

In other studies carried out by previous workers. S. aureus was reported to be sensitive to erythromycin and augmentin while resistance was recorded against tetracycline and ampicillin, although enhanced susceptibility has been reported by previous workers. The selection of antibiotic for use should be based on sensitivity testing. Administration of antibiotics to infected fish may increase severity of infection by converting local enteric infection into septicemia. It was however suggested that there is need for national antibiotic policy. Thus the study calls for stringent personal hygiene, environmental sanitation, good water source and clean hands before feeding the fish.

Table.1 Prevalenceof Bacterial species isolated from 220 fresh water fishes

\begin{tabular}{lccc}
\hline Bacterial species & No. of isolates & Total samples & \%Prevalence \\
\hline Escherichia coli & 16 & 44 & 36.36 \\
Proteus vulgaris & 10 & 44 & 22.70 \\
Salmonella typhi & 4 & 44 & 49.09 \\
Staphylococcus aureus & 4 & 44 & 18.18 \\
Staphylococcal epidermidis & 6 & 44 & 13.63 \\
Total & $\mathbf{4 4}$ & $\mathbf{2 2 0}$ & $\mathbf{1 0 0}$ \\
\hline
\end{tabular}

$\left(x^{2}=72.12 ; P<0.05\right.$ and $\left.P=0.00\right)$ 
Int.J.Curr.Microbiol.App.Sci (2017) 6(4): 2735-2743

Table.2 Morphological characterization of bacterial isolates from fishes

\begin{tabular}{llllll}
\hline $\begin{array}{l}\text { Probable } \\
\text { Isolate }\end{array}$ & $\begin{array}{l}\text { Color } \\
\text { characteristics }\end{array}$ & Margin & Elevation & Size \\
\hline & & & & & \\
\hline E. coli & Pink & Opaque & Irregular & Slightly elevated & Small \\
P.vulgaris & Brown & Opaque & Irregular & Elevated & Small \\
S. typhi & Dark & Opaque & Irregular & Flat & Big \\
S. aureus & Yellow & Translucent & Regular & Elevated & Small \\
S.epidermis & Pink & Opaque & Regular & Elevated & Small \\
\hline
\end{tabular}

Table.3 Biochemical characterization of the gram positive and gram negative bacterial isolates from fishes

\begin{tabular}{|c|c|c|c|c|c|c|c|c|c|}
\hline Isolates & Gram & Urease & Indole & Citrate & Catalase & $\mathbf{H}_{2} \mathbf{S}$ & G & Butt & Slant \\
\hline E. coli & - & - & + & - & + & - & + & $\mathrm{Y}$ & $\mathrm{Y}$ \\
\hline P. vulgaris & + & + & - & - & + & + & + & Y & $\mathrm{R}$ \\
\hline S.typhi & - & - & - & - & - & + & - & Y & $\mathrm{R}$ \\
\hline S.aureus & + & - & - & - & + & - & - & $\mathrm{N}$ & $\mathrm{R}$ \\
\hline S. epidermis & + & - & - & - & + & - & - & $\mathrm{N}$ & $\mathrm{R}$ \\
\hline
\end{tabular}

Key: TSI: Triple sugar iron test G: Gas. Y: Yellow. R: Red, $\mathrm{H}_{2} \mathrm{~S}$ : Hydrogen sulphide, +: Positive,-: Negative N: Red, Y: Yellow

Table.4 Antibiogram of fish bacterial isolates commonly used Antimicrobial agents (mcg)

\begin{tabular}{|c|c|c|c|c|c|c|c|c|c|c|}
\hline Isolates & OXF & CPX & $\mathbf{C H}$ & PEF & SP & AM & GN & TET & STM & SXT \\
\hline E. coli & 15 & 35 & 15 & 30 & 30 & 20 & 30 & 20 & 0 & 0 \\
\hline P. vulgaris & 52 & 0 & 0 & 20 & 15 & 20 & 1 & 5 & 0 & 0 \\
\hline S.typhi & 15 & 30 & 20 & 25 & 30 & 15 & 30 & 0 & 0 & 0 \\
\hline S.aureus & 27 & 52 & 92 & 75 & 16 & 16 & 15 & 2 & 5 & 0 \\
\hline S. epidermidis & 20 & 42 & 62 & 0 & 0 & 10 & 11 & 12 & 20 & 0 \\
\hline
\end{tabular}


Table.5 Dissipation of Antibiotic resistant patterns of the isolates to a variety of antimicrobial agents

\begin{tabular}{lcc} 
Resistant pattern & Isolates & Number of antibiotics \\
\hline STM, SXT & E.coli & 2 \\
STM, SXT, TET & S.typhi & 3 \\
STM, SXT, GN, CH & P. vulgaris & 4 \\
SXT, AM. & S.aureus & 2 \\
CPX, AM & S.epidermidis & 2
\end{tabular}

Key:S: $\quad$ Streptomycin STM, CotrimoxazoleSXT, AmoxicillinAM, Ciprofloxacin CPX, Tetracycline TET, Gentamycin GN, Chloramphenicol CH.

In conclusion, this study has exposed that some fresh water fishes in Nigeria harbors numerous microorganisms in their GIT which includes organisms such as E. coli, Proteus vulgaris, Salmonella typhi, Staphylococcus aureus, Staphylococcus epidermidis, as identified in our study. Their occurrence may be as a result of contaminated food source, health status and environmental risk factors. Ciprofloxacin showed highest susceptibility against the isolates, thus, emerging as the most effective antibiotic agent while septrin and streptomycin was the least susceptible antibiotic agent found in this study. The results of these study provided useful information on the occurrence and antibiotic susceptibility and resistant patterns of isolated organisms from the gastrointestinal tract of fish. This will help to prevent emergence of multidrug resistant bacteria.

\section{References}

Bauer, A.W., Kirby, W.M., Sherris, J.C. and Jurck, M. 1996. Antibiotic susceptibility testing by a standard disc method. American J. Clin. Pathol., 451: 493-496.

Ben Khemis, I., Audet, Fournier, R. and De la Noue, U. 2003. Early weaning of winter flounder

(Pseudopleuronectes americanus Walbaum) larvae on a commercial microencapsulated diet. Aquaculture Res., 34: 445-452.
Bergey's, D.H. 1992. Manualof Determinative Bacteriology. Seventh Edition. The Williams and Wilkins Company, Baltimore, 336-583.

Birkbeck, T.H. and Verner-Jeffreys, D.W. 2002. Development of the intestinal microflora in early life stages of flatfish, 149-160.

Cheesbrough, M. 2005. District laboratory practice in tropical countries. ECBS Cambridge University Press, 2: 97-182.

Collinder, E., Bjornhag, G., Cardona, M., Norin, E., Rehbinder, C. and Midtvedt, T. 2003. Gastrointestinal host-microbial interactions in mammals and fish: Comparative studies in man, mice, rats, pigs, horses, cows, elk, reindeer, salmon and cod. Microbial Ecol. Health Dis., 15: 66-78.

Egah, D.Z., Bello, C.S. and Betal, S. 1998. Antimicrobial susceptibility pattern of S. aureus in Jos, Nigeria. J. Med., 8(2): 5860.

Evans, J.R., Doyle, J., Dolores, G. and Evans, J. 2007. "Escherichia coli". Med. Microbiol., the original on 2007-11-02.

FAO. 2007. The State of World Fisheries and Aquaculture, Food and Agriculture Organization, United Nations, Rome, Italy, 22-25.

Gomathi, M., Dillarani, V. and Nithya, G. 2016. Characterization and antimicrobial susceptibility patterns of non fermenting Gram negative bacilli from various 
clinical samples in tertiary care hospital. Indian J. Microbiol. Res., 3(4): 387-391.

Hansen, G.H. and Olafsen, J.A. 1999. Bacterial interactions in early life stages of marine cold water fish. Microbial Ecol., 38: 1-26.

Huber, I., Spanggaard, B., Appel, K.F., Rossen, L., Nielsen, T. and Gram, L. 2004. Phylogenetic analysis and in situ identification of the intestinal microbial community of rainbow trout (Oncorhynchusmykiss, Walbaum). J. Appl. Microbiol., 96: 117-132

Ikegwu, I.J., Amadi, E.S. and Iroha, I.R. 2008. Antibiotic sensitivity pattern microorganisms in Abakaliki, Nigeria. Pak. J. Med. Sci., 2(24): 231-235.

Kanika, S. 2007. Manual of microbiology tools and techniques. ${ }^{\text {nd }}$ (Edition). Ane books private limited New Delhi, 2007: 86-88.

Olafsen, J.A. 2001. Interactions between fish larvae and bacteria in marine aquaculture. J. Aquaculture, 233-247.
Olayemi, A.B. and Oyagede, J.S.O. 1997. Incidence of antibiotic resistance among Escherichia coli isolated from clinical and river water Nigeria. Med. J., 17(4): 207-209.

Ringo, E. and Birkbeck, T.H. 1999. Intestinal microflora of fish larvae and fry. Aquaculture Res., 30: 73-93.

Spanggaard, B., Huber, I., Nielsen, J., Nielsen, T., Appel, K.F. and Gram, L. 2000. The microflora of rainbow trout intestine: acomparison of traditional and molecular identification. Aquaculture, 182: $1-15$.

Trust, T.J. and Sparrow, R.A.H. 1974. The bacterial flora in the alimentary tract of fresh water Salmonid fishes. $J$. Microbiol., 20: 1219-1222.

WHO. 2006. Report of a joint FAO/OIE/WHO expert consultation on antimicrobial use in aquaculture and antimicrobial resistance: Seoul, Republic of Korea, 13-16.

\section{How to cite this article:}

Mailafia Samuel and Anjorin Samuel Toba. 2017. Occurrence and Antimicrobial Susceptibility Pattern of Bacteria Isolated from Gastrointestinal Tract of Fresh Water Fishes in Abuja, Nigeria. Int.J.Curr.Microbiol.App.Sci. 6(4): 2735-2743. doi: http://dx.doi.org/10.20546/ijcmas.2017.604.317 